\title{
Better Normal, A Silver Lining in 2020: JAFES is Accepted for Indexing in PubMed Central
}

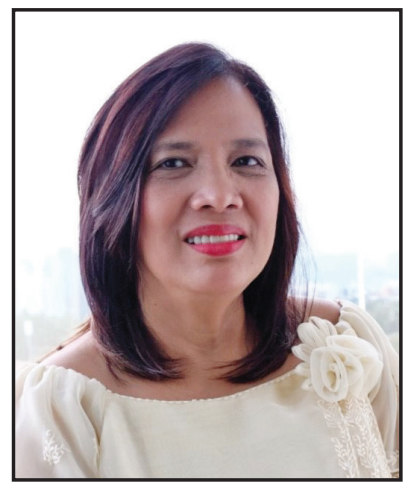

COVID-19 continues to redefine the way we live and the way we work. We have no recourse but to embrace a different way of life: wearing masks and face shields in public, establishing physical and social distancing, and practicing hand hygiene at all times. As medical practitioners, educators, and researchers, we adapt our profession and practice in the context of this public health threat.

We have realized that there are novel and innovative ways to take care of our patients, confer and meet with our colleagues, teach our students, and mentor our Residents and Fellows. When it concerns our researches for purposes of understanding a disease and improving a policy, data gathering among patients in clinics and hospitals remains limited; yet different research designs and strategies still enable meaningful studies.

For us at the JAFES, the new normal meant getting together virtually for the bi-annual editorial board meeting. To our pleasant surprise, the virtual meetings allowed for near complete attendance by the Philippine team as well as the ASEAN editors. With live interactive discussions, even if remote, the outputs became more profound and prolific. (Figure 1). Why didn't we think of virtual meeting before the pandemic? Technology allowed us to see each other beyond the usual written correspondences. Despite the challenges of bandwidth and connectivity, we had in-depth discussions on concerns with publication ethics and operational issues.

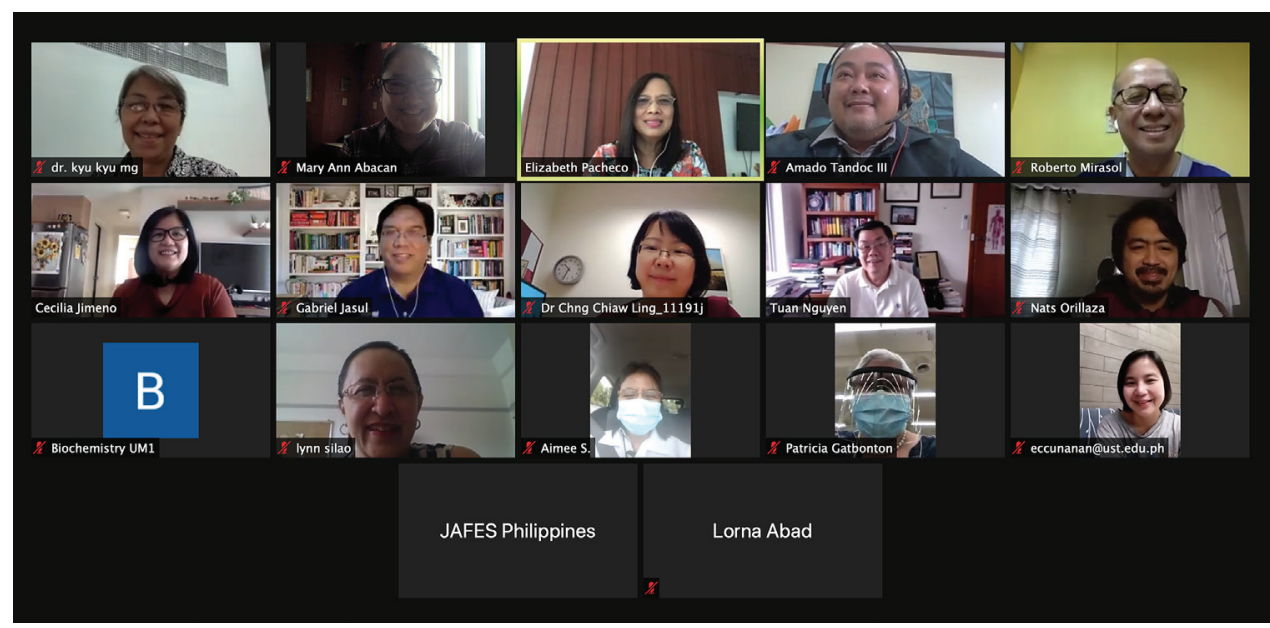

Figure 1. JAFES Editorial Board members from the ASEAN countries hold a productive virtual editorial board meeting to finalize its second issue.

The May JAFES issue headlined diabetes, its impact on one's susceptibility to COVID-19 and likelihood of having a more stormy course. This time, the November issue banners a review article on COVID-19 and thyroid disorders, underscoring the need for re-assessing how we take care of endocrine disorders during these challenging times. Recognizing some delays in the patients' trips to the hospital or clinic for non-urgent elective care, periodic follow-ups of these conditions can still be successfully carried out through tele consults with electronic prescriptions. Patient education continues through the electronic sharing of materials and videos. When necessary, we have designated smaller teams with safe hospital set-ups and technologies to carry out the task, all for the best interests of our patients to proceed with diagnostic work-ups, elective surgeries, and other treatment options. The internet has become a more powerful tool; and, as most everyone is engaged in its use, guidance for its more responsible use should become available. 
Amid the uncertainties and challenges brought on by the COVID-19 pandemic, we celebrate another major milestone in the continuing journey of the JAFES. We formally announce here our acceptance to PubMed Central, (Figure 2), after being included in Scopus and Clarivate Analytics Emerging Sources Citation Index in the last 2 years. Launched in 2000, PubMed Central is a free archive of full-text biomedical and life sciences journal articles, serving as a digital counterpart to the print journal collection of the US National Library of Medicine. As a participating journal, JAFES shall be depositing full text articles starting from 2017 and these shall be available 100\% open access and searchable also in MedLine.

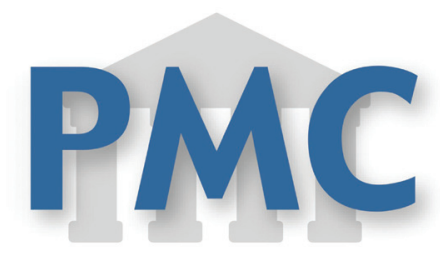

\section{US National Library of Medicine National Institutes of Health}

Figure 2. JAFES passed the scientific quality and technical review by NML for PMC.

The pandemic shook us out of our comfort zones, obliged us a new look at doing things, and led us to improve our ways and the effects on the people that we serve. We all look toward a better normal.

We wish everyone a safe and healthy end of 2020 and hope for a better New Year 2021!

Elizabeth Paz-Pacheco

Editor-in-Chief

https://doi.org/10.15605/jafes.035.02.15 